\title{
A Fault Diagnosis Method Based on Improved Pattern Spectrum and FOA-SVM
}

\author{
Dejian Sun, Bing Wang, Xiong Hu and Wei Wang \\ Logistics Engineering College, Shanghai Maritime University, 201306, ShangHai, China.
}

(Received 30 November 2017; accepted 22 November 2018)

\begin{abstract}
A fault diagnosis method using improved pattern spectrum $(I P S)$ and $F O A-S V M$ is proposed. Improved pattern spectrum is introduced for feature extraction by employing morphological erosion operator, and this feature is able to present fault information for roller bearing on different scales. Simulation analysis is processed and shows that, the value of $I P S$ has a steady distinction among different fault types and the calculating amount is less than traditional method. After feature extraction, $S V M$ with $F O A$, which can help with seeking optimal parameters, is employed for pattern recognition. Experiments were conducted, and the proposed method is verified by roller bearing vibration data including different fault types. The classification accuracy of the proposed approach on training is $87.5 \%\left(\frac{21}{24}\right)$ and reaches $91.7 \%\left(\frac{44}{48}\right)$ in a testing data set. The analysis shows that the method has a good diagnosis effect and an acceptable recognition result.
\end{abstract}

\section{INTRODUCTION}

A roller bearing is a key mechanical component. It is meaningful to propose an efficient fault diagnosis technique for its reliability. Vibration signal processing is widely used for the reason that vibration signals are sensitive to structural dynamics condition variation. ${ }^{1}$ Therefore, it is feasible to excavate faulty features in the vibration signals with efficient techniques. Three basic process of diagnosis technique contains data collection, feature extraction and diagnostic methods. ${ }^{2}$

As to the step of feature extraction, when roller bearings are in the running condition, the non-liner feature is distinct because of some non-liner factors. ${ }^{3}$ For this reason, the linearbased processing methods are not able to analyze working conditions accurately. Considering the non-linear feature of the vibration signal, some non-linear analytical techniques offer a feasible way in processing the non-liner characteristics of a roller bearing, such as information entropy, fractal dimensions, and so on.

Among the non-linear analytical techniques, the mathematical morphological particle analysis is able to describe the shape changing information on different scales, it is apt to analyze the essence of signal on different levels., ${ }^{4,5}$ This method is widely used in the area of image feature description, image segmentation, and image restoration. ${ }^{6-8}$ There are some studies in fault feature extraction of mechanical signals. ${ }^{9,}{ }^{10}$ Considering a poor effect existing in fault diagnosis with pattern spectrum $(P S)$, a morphological erosion operator is introduced in the mathematical morphological particle analysis. A definition of improved pattern spectrum $(I P S)$ is proposed. Considering the advantages of this method in feature description, IPS is proposed for the feature extraction.

As to the step of diagnostic method, it is significant after selecting $I P S$ in feature extraction. A typical artificial neural network $(A N N)$ needs sufficient samples, which are not prac- tical. ${ }^{11}$ Taking statistical learning theory as the basis, support vector machines $(S V M)$ are able to obtain an optimal solution for local and global. ${ }^{12}$ For the advantages in high accuracy and smaller sample number, $S V M$ is brought into machines fault diagnosis. ${ }^{13-15}$ It is researched that parameters selection influence the effect of $S V M$. In order to get an optimal combination, fruit fly optimization algorithm $(F O A)$ is brought in parameters optimizing of $S V M$ in this paper.

In conclusion, a diagnostic method is developed based on improved pattern spectrum and $F O A-S V M$. The method of $I P S$ brought in is feature extraction, and $S V M$ with FOA is chosen as a diagnostic model. First, a vibrating signal is analyzed based on the IPS method and forms an efficient feature set. Then, five statistic parameters are calculated as the feature set. Finally, the five-dimension feature is input to $S V M$ and the parameters are optimized with $F O A$. Fault types can be identified with this method. The proposed method is verified by roller bearing vibration data including different fault types.

The paper is organized as follows: Section 2 briefly introduces pattern spectrum. A definition of IPS is proposed in Section 3. In Section 4, fault diagnosis flow using $I P S$ and $S V M$ is concluded, and in Section 5, the proposed method is verified, and the results are discussed. Finally, the conclusion of this paper is given in Section 6.

\section{PATTERN SPECTRUM THEORY}

Pattern spectrum is based on mathematical morphological particle analysis, which is an effective theory in dealing with image granularity and shape features. The basic thought is to analyze images using structuring elements with different size and shape, getting internal characteristics of the images finally.

Mathematical morphological particle analysis is defined as a set of image transformation as $\Psi=\left\{\psi_{\lambda}\right\} \lambda \geq 0$, which should satisfy the following three conditions: ${ }^{4}$ 


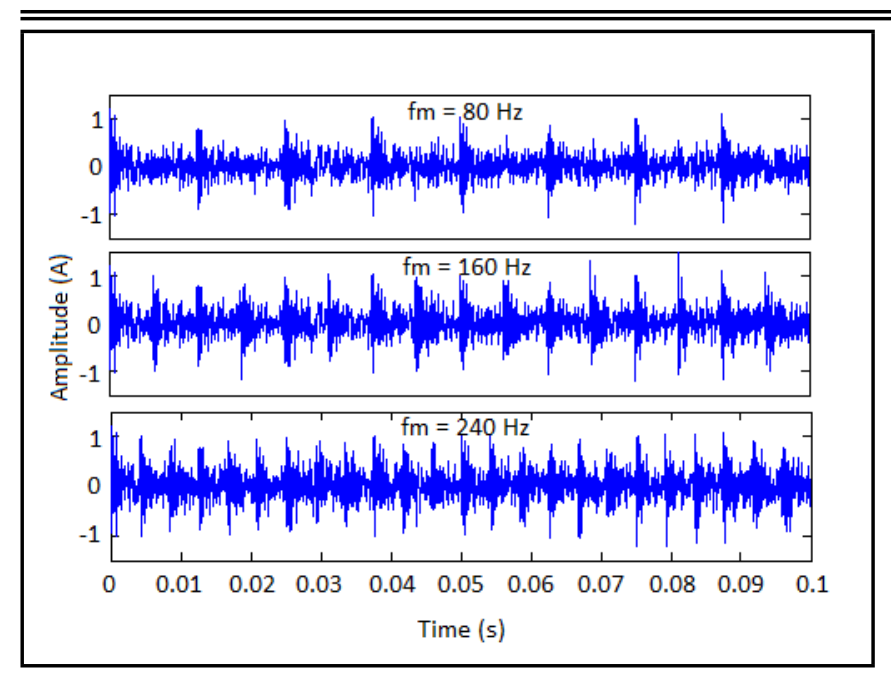

Figure 1. Time domain of simulation signal.

(1) Monotonically increasing.

$\forall \lambda \geq 0, X \subset Y \Leftrightarrow \psi_{\lambda}(X) \leq \psi_{\lambda}(Y)$.

(2) Non-epitaxial. $\forall \lambda \geq 0: \psi_{\lambda}(X) \leq X$.

(3) $\forall \lambda, \mu \geq 0$,

$\left.\psi_{\mu}\left(\psi_{\lambda}(X)\right)=\psi_{\lambda}\left(\psi_{\mu}(X)\right)=\psi_{\max (\lambda, \mu)}(X)\right)$.

The mathematical opening operator $\circ$ is able to meet the above three conditions, therefore, mathematical morphological particle analysis is usually applied based on this operator. Assuming that $g$ is a unit structuring element, and $\lambda$ is the scale. Mathematical morphological particle analysis is defined as:

$$
\psi(X)=X \circ \lambda g, \lambda g=g \oplus \ldots \oplus g
$$

Pattern spectrum $(P S)$ is introduced based on the definition above, and it is a series of curves presenting mathematical particle size distribution.

Supposing $f$ is the time domain signal and $g$ is the unit structuring element, pattern spectrum for $f$ on scale $\lambda$ can be defined as the following formula:

$$
P S(f, \lambda, g)=\left\{\begin{array}{ll}
-\frac{d A(f \circ \lambda g)}{d \lambda} & \text { if } \lambda \geq 0 \\
-\frac{d A(f \bullet(-\lambda) g)}{d \lambda} & \text { if } \lambda<0
\end{array}\right. \text {. }
$$

In the formula above, $A(f)$ means the coverage for $f$ in the definition domain. A mathematical opening operator is introduced as $\lambda \geq 0$, and the $P S$ value represents the structuring information. Relatively, mathematical closing operator is introduced as $\lambda<0$ and the $P S$ value means the background information. The plus and minus section for $P S$ has consistency and $P S$ with $\lambda \geq 0$ is usually introduced for analysis, the paper is also in this case.

When analyzing one-dimension discrete signal, the scale is continuous integer and the definition of $P S$ can be simplified as the formula Eq. (3) (see top of the next page). As to the nonepitaxial feature for morphological opening operator, the $P S$ of a discrete signal is a set of non-negative number sequences.

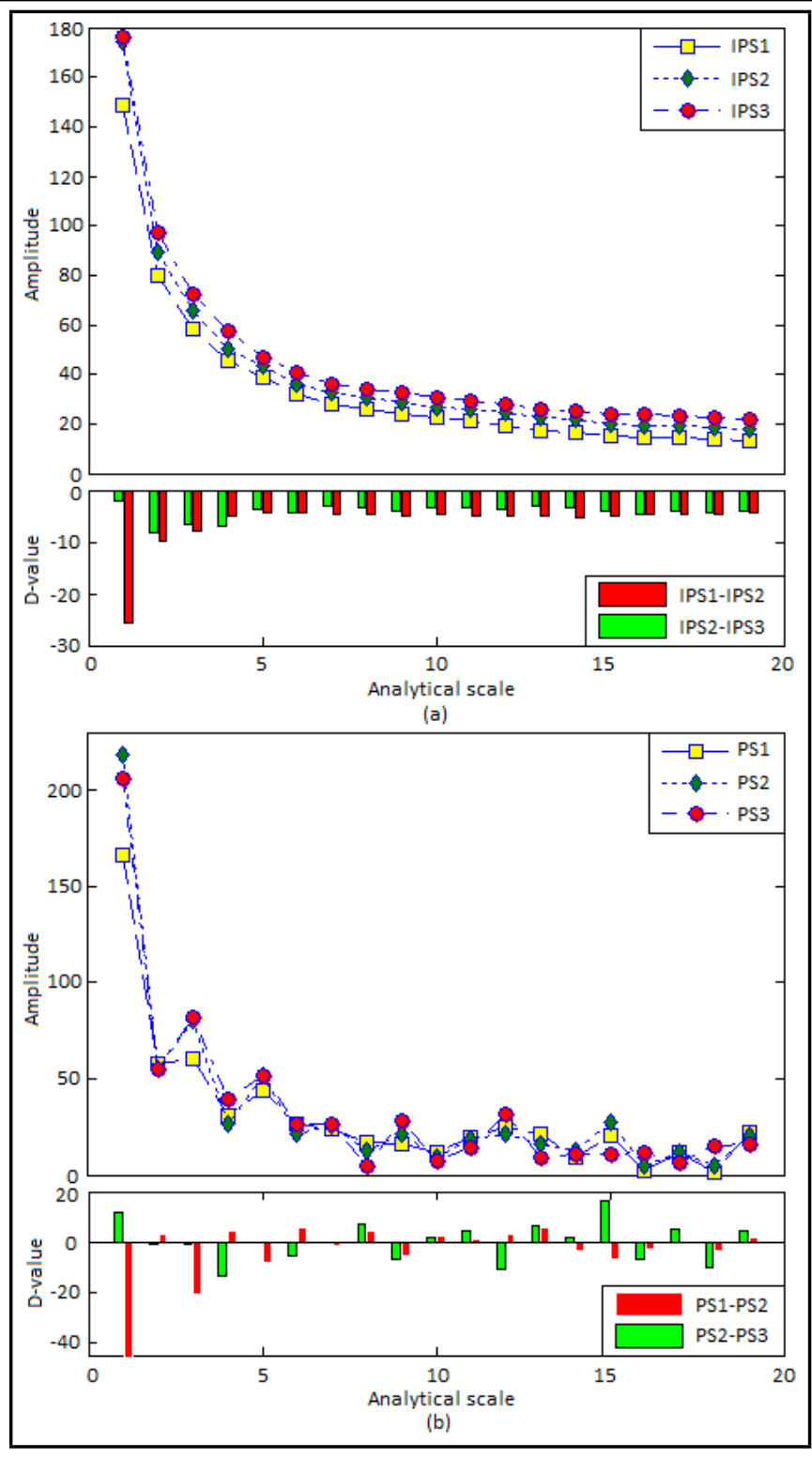

Figure 2. $I P S$ and $P S$ curves with D-value of different feature frequency. (a) $I P S$ curves, (b) $P S$ curves.

\section{PROPOSITION OF IPS}

\subsection{Definition of Improved Pattern Spectrum}

Considering the effect in fault feature extraction, the morphological erosion operator $\Theta$ is introduced and proposes a definition of improved pattern spectrum $(I P S)$. The definition are as follows:

$$
I P S(f, \lambda, g)=\left\{\begin{array}{ll}
-\frac{d A(f \Theta \lambda g)}{d \lambda} & \text { if } \lambda \geq 0 \\
-\frac{d A(f \oplus(-\lambda) g)}{d \lambda} & \text { if } \lambda<0
\end{array}\right. \text {. }
$$

In the formula above, the mathematical erosion operator is introduced as $\lambda \geq 0$ where the mathematical dilation operator is introduced as $\lambda<0$. Considering the consistency for the plus and minus section, the $I P S$ in the plus section is usually introduced for analysis. 


$$
\left\{\begin{array}{ll}
P S_{+}(\lambda, g)=A[f \circ \lambda g-f \circ(\lambda+1) g] & \text { if } 0 \leq \lambda \leq \lambda_{\max } \\
P S_{-}(\lambda, g)=A[f \bullet(-\lambda) g-f \bullet(-\lambda+1) g] & \text { if } \lambda_{\min } \leq \lambda \leq 0
\end{array} .\right.
$$

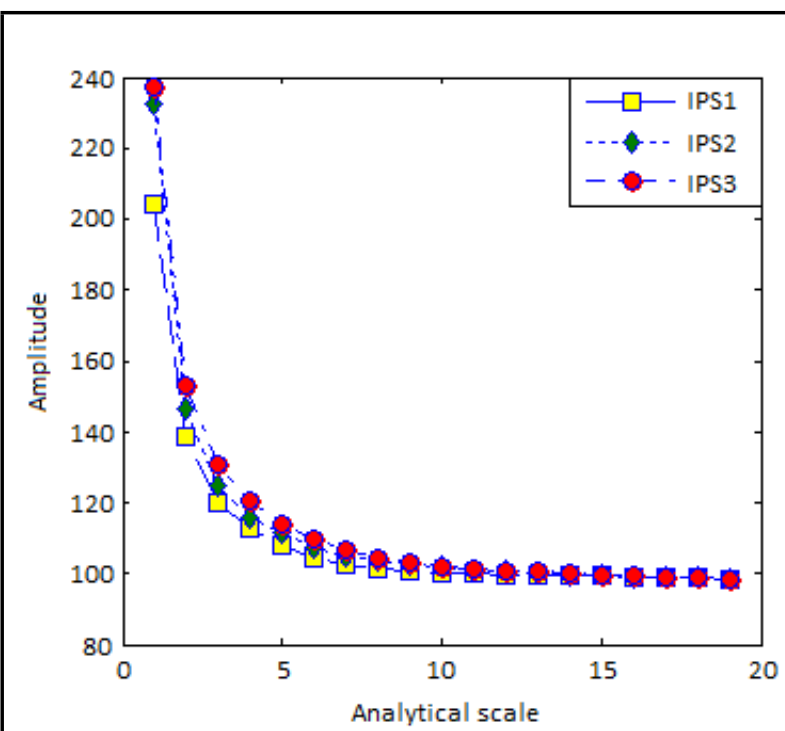

(a)

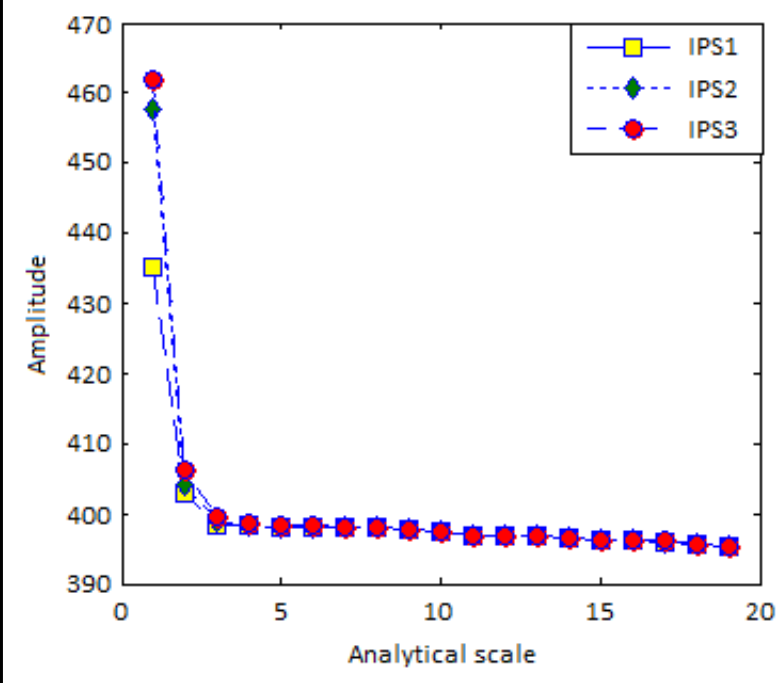

(b)

Figure 3. IPS curves based on triangular structuring element with different amplitude. (a) $g=\left[\begin{array}{lll}0 & 0.05 & 0\end{array}\right]$, (b) $g=\left[\begin{array}{lll}0 & 0.2 & 0\end{array}\right]$.

The definition of IPS can be simplified as the formula Eq. (5) (see top of the next page) when analyzing onedimension discrete signal. The scale is defined as a continuous integer, and the IPS value is a set of non-negative number sequences.

\subsection{Simulation and Analysis}

A roller bearing simulation model is built in the following formula according to the operating and failure mechanism: ${ }^{16}$

$$
y(k)=e^{-\alpha t} \sin 2 \pi f_{c} k T, \quad t=\bmod \left(k T, \frac{1}{f_{m}}\right) .
$$

In the formula above, parameters of $\alpha, f_{m}, f_{c}, T$ represent the exponential frequency, modulation frequency, carrier frequency, and sampling interval respectively. In the simulation,

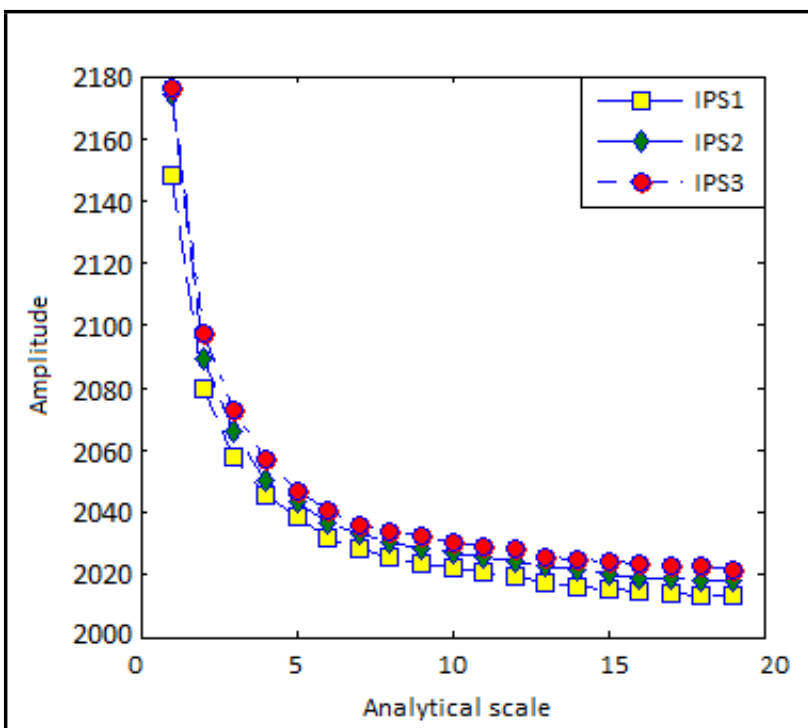

(a)

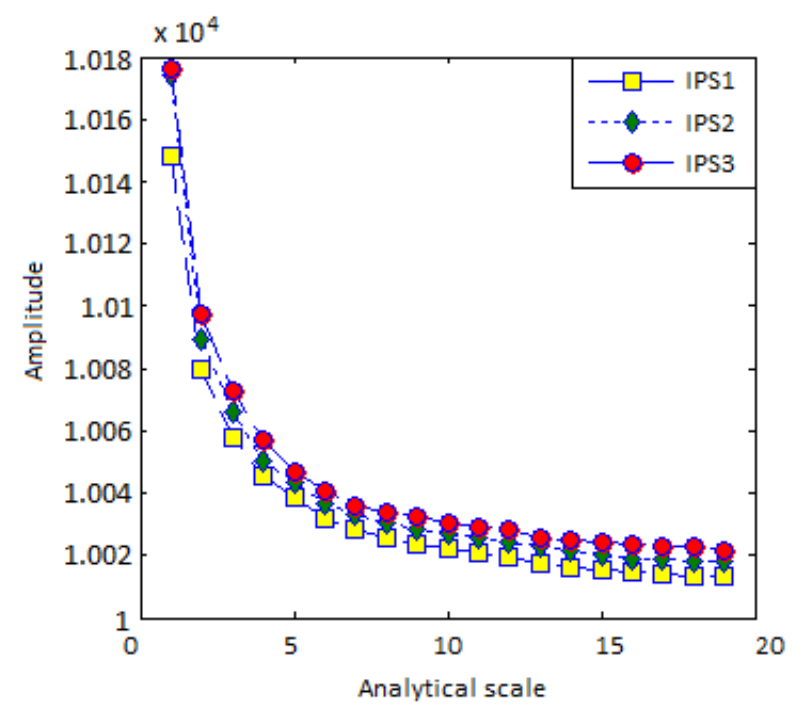

(b)

Figure 4. $I P S$ curves based on triangular structuring elements with different amplitudes. (a) $g=\left[\begin{array}{lll}1 & 1 & 1\end{array}\right]$, (b) $g=\left[\begin{array}{lll}5 & 5 & 5\end{array}\right]$.

$\alpha$ is set as 800 and $f_{c}$ is set as $5000 \mathrm{~Hz}$. The sample time is 0.1 second and $T$ is set as $\frac{1}{20000}$. Gaussian white noise is added in the simulation signal to accord with reality. Modulation frequency $f_{m}$ is set as $60,120,240$ in order to simulate different fault types for bearings respectively. Three simulation signals with different modulation frequencies are shown in Fig. 1.

As to analyzing with $I P S$, the unit structuring element is set as $g=\left[\begin{array}{lll}0 & 0 & 0\end{array}\right]$, and the maximum analytical scale is set as $\lambda_{\max }=20$. The results are shown in Fig. 2a where IPS1$I P S 3$ correspond three signals with $f_{m}$ equals 80,160 , and 240 respectively. Three curves present a monotone decreasing trend with the increasing analytical scale. The $\mathrm{D}$-values among the three curves are shown in the histogram; the D-values are all minus on the scales and present a steady distinction of dif- 


$$
\left\{\begin{array}{ll}
I P S_{+}(\lambda, g)=A[f \Theta \lambda g-f \Theta(\lambda+1) g] & \text { if } 0 \leq \lambda \leq \lambda_{\max } \\
I P S_{-}(\lambda, g)=A[f \oplus(-\lambda) g-f \oplus(-\lambda+1) g] & \text { if } \lambda_{\min } \leq \lambda \leq 0
\end{array} .\right.
$$

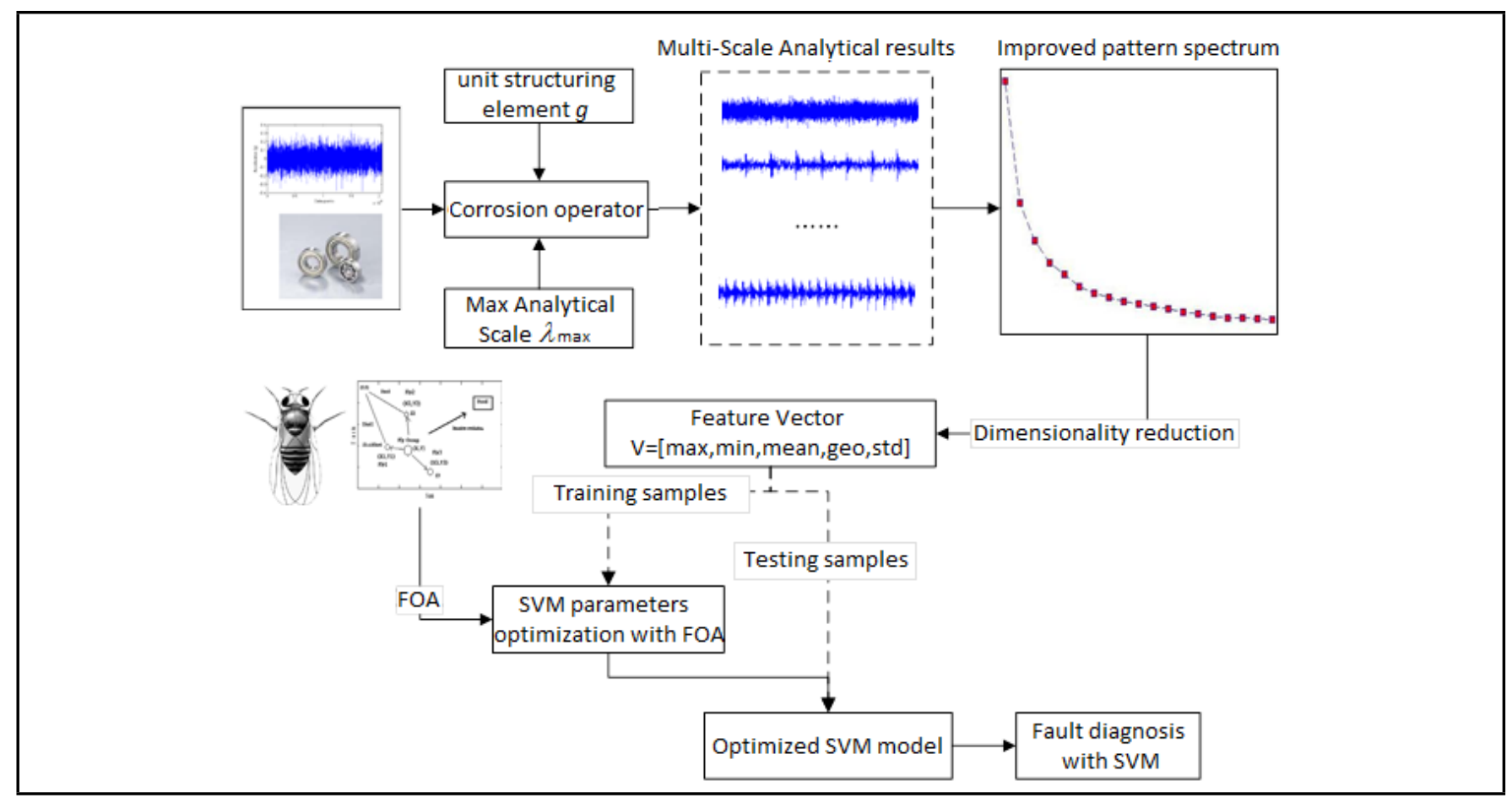

Figure 5. Flow of diagnosis based on $I P S$ and $S V M$ with FOA.

ferent simulation faults.

Compared to the traditional pattern spectrum method, the calculated three $P S$ curves are shown in Fig. $2 \mathrm{~b}$ with the same unit structuring element and maximum analytical scale. As can be seen, there is no monotone trend with the increasing of analytical scale, the D-values between the three curves are not consistent, presenting a bad distinction of different simulation faults.

In addition, considering that morphological opening operation is defined as the sequential execution of morphological corrosion and dilation operation, the proposed method based on IPS has less time consumption than the traditional PS method.

A different structuring element type has an influence on the IPS result. The calculating result with triangular unit structuring element is shown in Fig. 3. The simulation signal and maximum analytical are the same as above. It can be seen that the distinction effect becomes worse with the increasing amplitude of triangular structuring element. It is clear that there is no distinction when the analytical is greater than five in Fig. 3b.

The calculating result with rectilinear structuring elements are shown in Fig. 4. It can be seen that there is no apparent effect distinction between $g=\left[\begin{array}{lll}1 & 1 & 1\end{array}\right]$ and $g=\left[\begin{array}{lll}5 & 5 & 5\end{array}\right]$. Therefore, structuring the element as $g=\left[\begin{array}{lll}0 & 0 & 0\end{array}\right]$ is a good choice considering the calculation amount and effect.

\section{FAULT DIAGNOSIS FLOW USING IPS AND FOA-SVM}

A fault diagnosis method using IPS and FOA-SVM is proposed in this section, the flow is shown in Figure 5. First, we must choose the unit structuring element as well as the maximum analytical scale; calculate features based on IPS; and then, in order to reduce the dimensions of IPS curve, calculate five statistic parameters and constitute the feature vectors, which include the maximum value, the minimum value, the average value, the geometric mean, and the standard deviation. On this basis, $F O A$ (fruit fly optimization algorithm) ${ }^{17-19}$ is introduced in $S V M$ parameters optimization using feature vectors of training samples. Finally, the fault types will be diagnosed by the optimized $S V M$ model.

\section{APPLICATION AND DISCUSSION}

\subsection{Data introduction}

The bearing vibration data was employed from CWRU. ${ }^{20,21}$ The experimental setup is shown in Fig. 6. The chosen vibration data contains four fault types under the load of $0.746 \mathrm{kw}$ are shown in Fig. 7.

A description of the bearing data sets are listed in Table 1, which are divided into training and testing samples with 1:2 proportion randomly. The four types are labeled from 1 to 4 .

\subsection{Feature extracting based on IPS}

$I P S$ is employed in feature extracting in this section, the maximum analytical scale is set as $\lambda_{\max }=20$, and the unit 


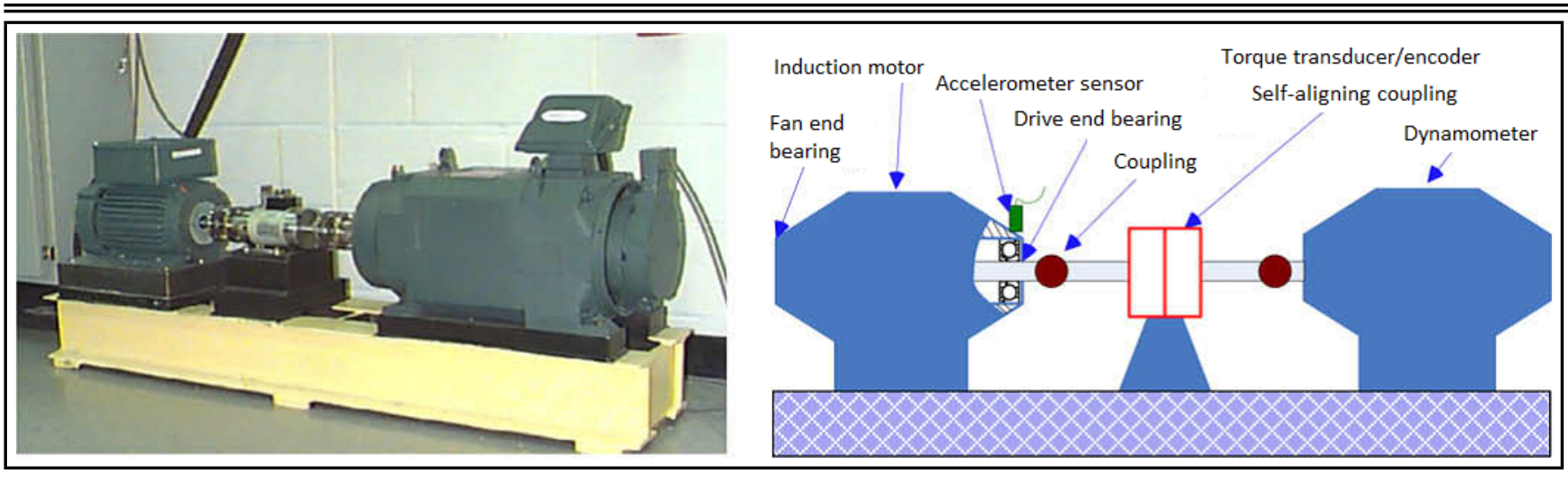

Figure 6. The experimental setup for bearing. (a) Experimental setup, (b) Diagram of the experimental setup.

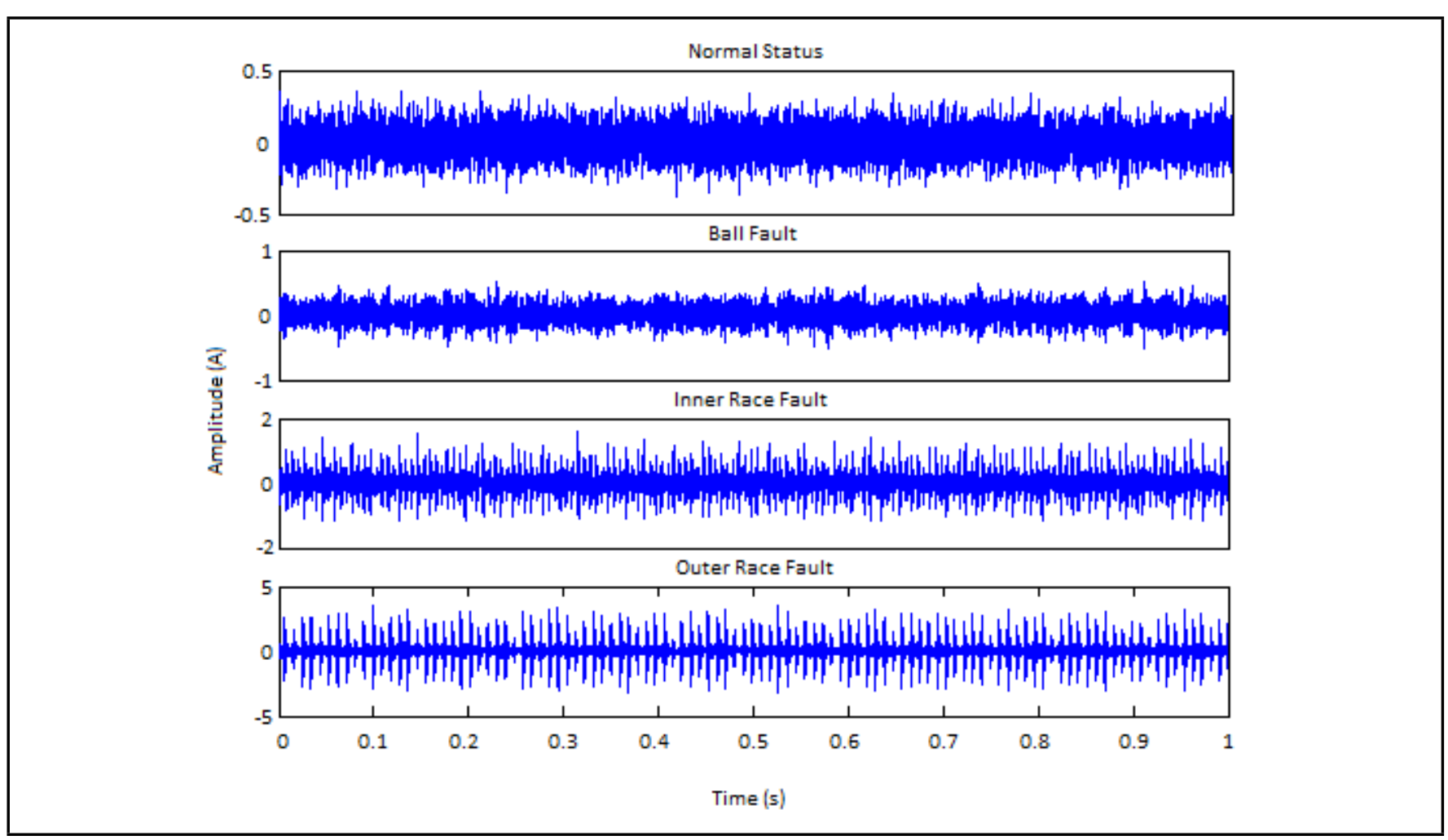

Figure 7. Time-domain waveform of the four types.

structuring element is $g=\left[\begin{array}{lll}0 & 0 & 0\end{array}\right]$. Calculating $I P S$ for training samples and the $I P S$ curves are shown in Fig. 8. Evident distinction exists among different conditions, and the IPS curves contain information for each condition.

Values of the four groups of typical samples are shown in Fig. 9. It is clear that the $I P S$ curve presents a declining trend along with the increasing analytical scale. Considering the distinction on the IPS value, feature extraction based on IPS has a good effect compared to the $P S$ curves in Fig. 10.

The IPS calculated above have nineteen dimensions that will increase computational complexity for the $S V M$ model. Five statistic parameters are calculated in order to reduce the dimensions, as shown in Fig. 11.

\subsection{Pattern classification using FOA-SVM}

The five dimension features calculated above are used to train $S V M$, and FOA is introduced to get the optimal param- eters of $S V M$, including penalty factor and kernel function parameter $y$.

In the training course with $F O A$, the iteration generation is set as 100 and the fruit fly group is set as 20 . The classification accuracy for training features is set as the aiming function. After the optimization, the best value reaches $97.83 \%$, and the optimal penalty parameter $C=0.5642$, kernel parameter $y=2.9129$.

After training with $F O A$, the testing feature sets are input to the $S V M$ model; the output is shown in Fig. 12. The misclassified samples include three in the training and four in the testing. The whole classification accuracy of the proposed approach on training is $87.5 \%\left(\frac{21}{24}\right)$ and reaches $91.7 \%\left(\frac{44}{48}\right)$ in testing data set. The result indicates the effectiveness and suitability of $I P S$ as feature vectors.

A typical $B P$ neural network is introduced for comparison. The structure is $5 \times 15 \times 1$, and the training and testing samples 
Table 1. Description of bearing data set.

\begin{tabular}{||c|c|c|c|}
\hline Bearing condition & Number of training samples & Number of testing samples & Label of classification \\
\hline Good condition & 6 & 12 & 1 \\
\hline Roller element fault & 6 & 12 & 2 \\
\hline Inner race fault & 6 & 12 & 3 \\
\hline Outer race fault & 6 & 12 & 4 \\
\hline
\end{tabular}

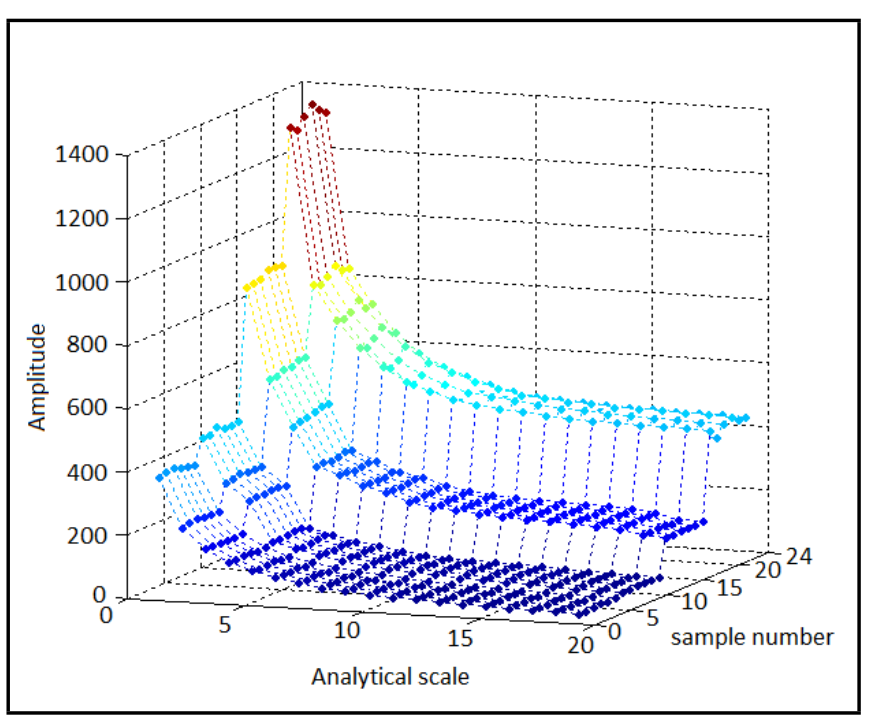

Figure 8. IPS curves distribution for training sample.

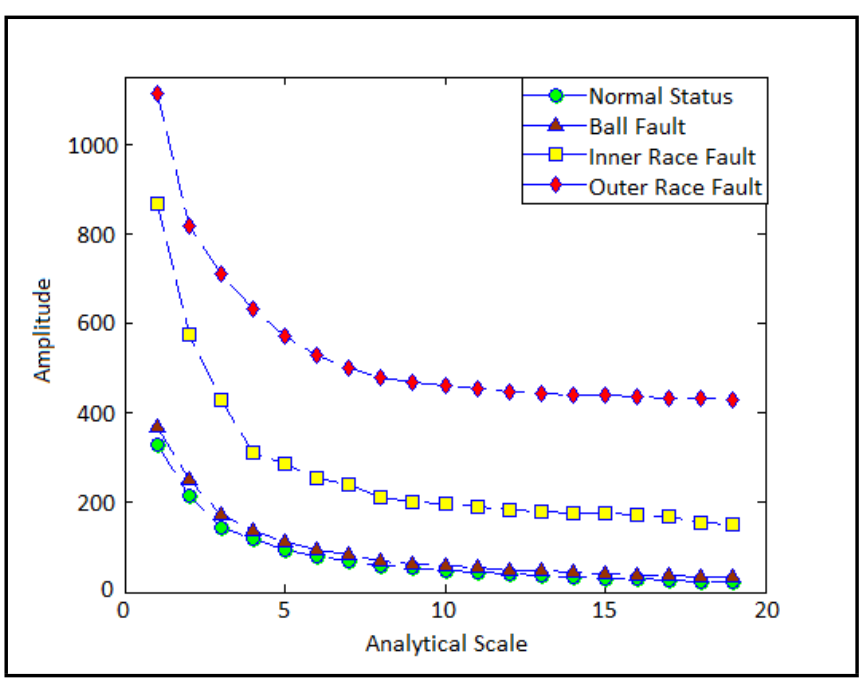

Figure 9. IPS curve of typical training sample.

have no differences with $S V M$. The recognition result of $B P$ network is shown in Fig. 13. Five samples are misclassified in training, and the training classification accuracy reaches $79.2 \%$ $\left(\frac{19}{24}\right)$. Furthermore, eight samples are misclassified in the testing and the testing classification accuracy reaches $81.8 \%\left(\frac{36}{44}\right)$. This comparison presents the advantage of $S V M$ with fruit fly optimization algorithm.

\section{CONCLUSIONS}

In this paper, a morphological erosion operator is introduced to the mathematical morphological particle analysis, and a fault diagnosis method using improved pattern spectrum and $F O A-S V M$ is proposed. Experiments were conducted, and the proposed method was verified by roller bearing vibration

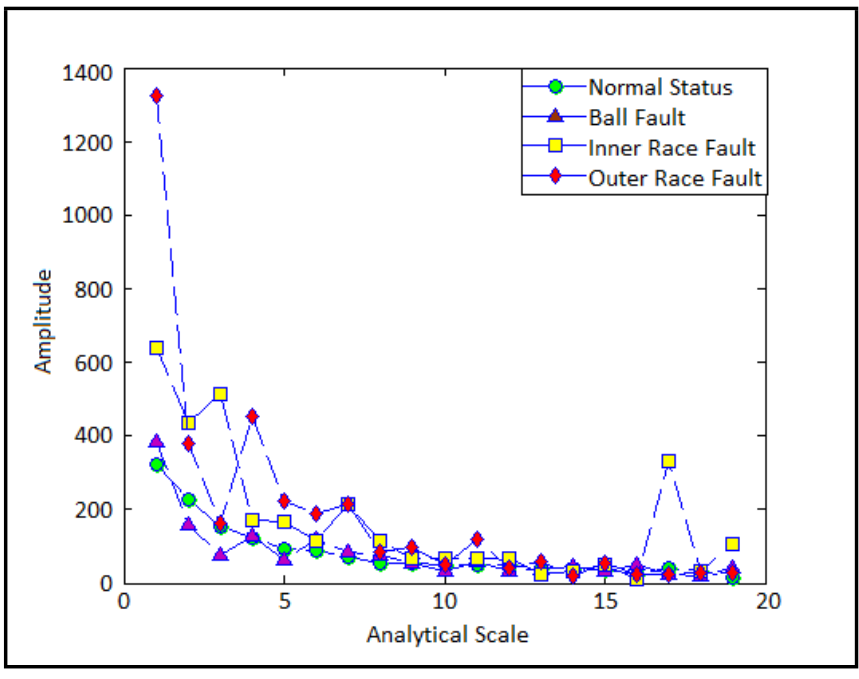

Figure 10. $P S$ curve of typical training sample.

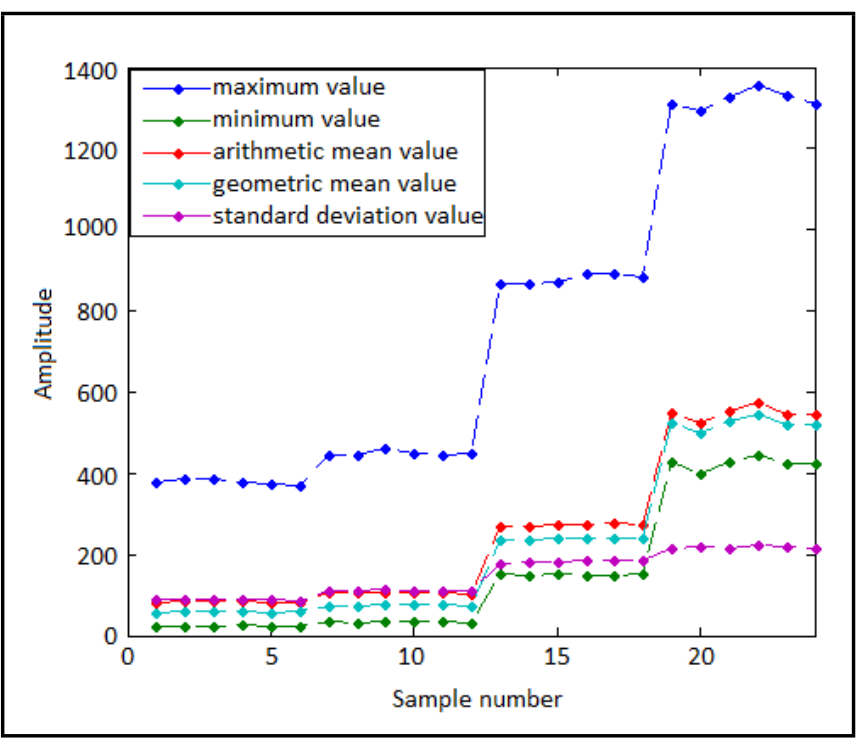

Figure 11. Five statistics of the $I P S$.

data including different fault types. Some conclusions of the study are as follows:

(1) Morphological erosion operation has different operation character compared with morphological opening operator. $I P S$ based on erosion operator is able to present morphological characters of signals on different scales, leading to a better effect in distinguishing fault types.

(2) Considering that morphological opening operation is defined as the sequential execution of morphological corrosion and dilation operation, the proposed method based on IPS has less time consumption and a better effect than traditional $P S$ methods.

(3) Considering that morphology operation involves only simple addition and subtraction, the proposed IPS method has 


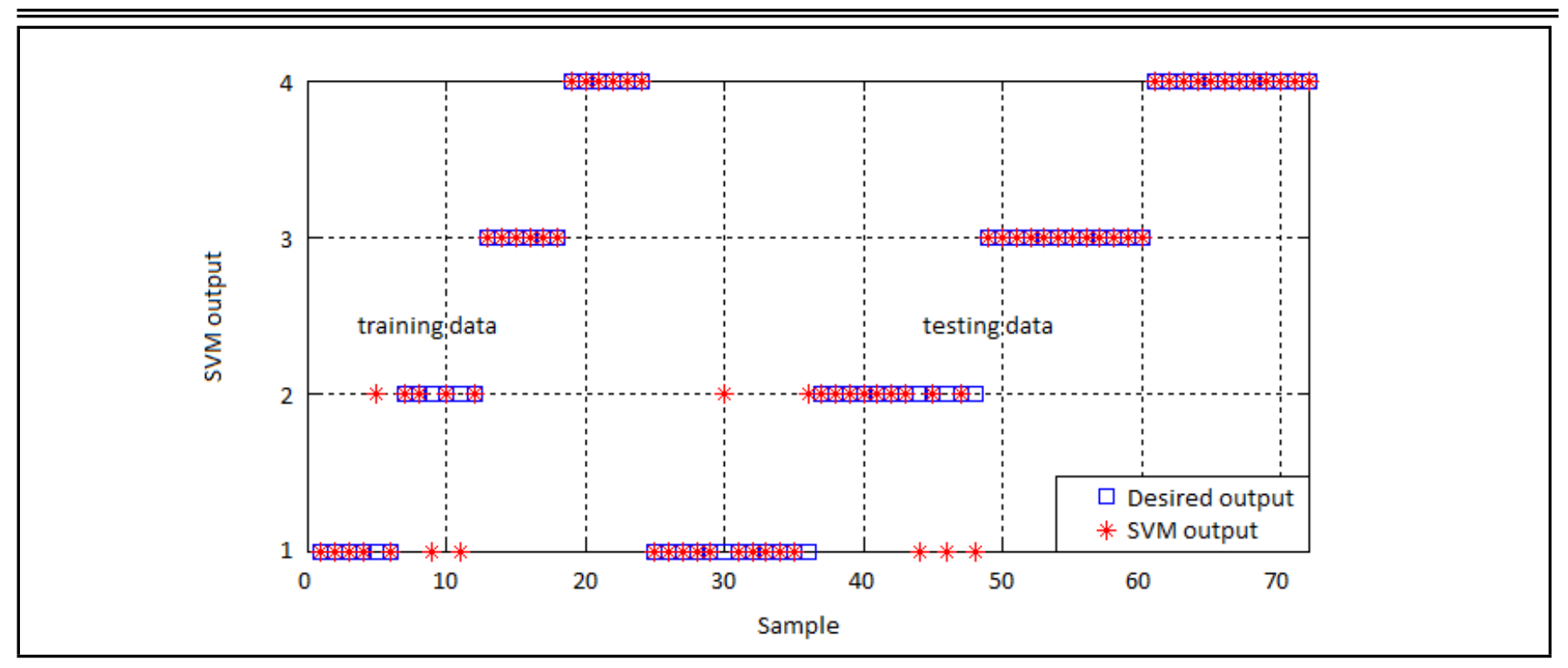

Figure 12. The classification output using the proposed approach.

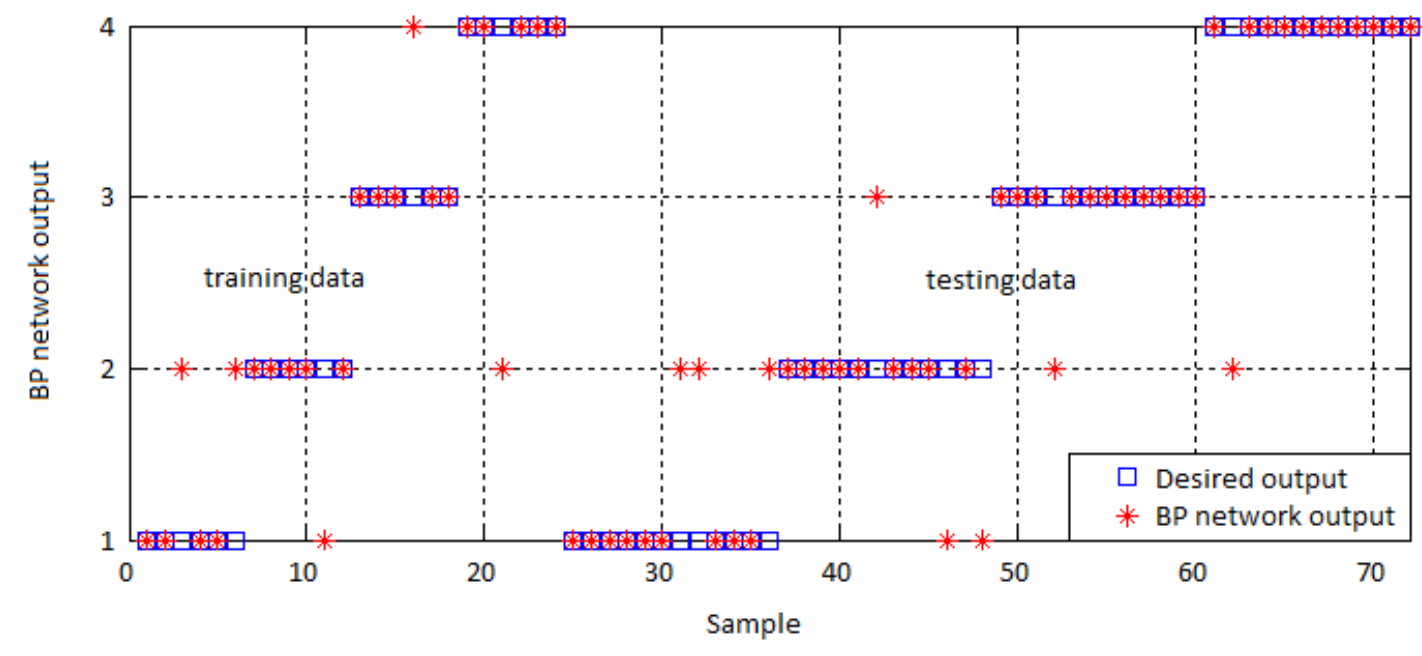

Figure 13. The classification output using $B P$ network.

a small calculation amount and high efficiency, it is promising to introduce this method to on-line monitoring processing systems.

(4) $B P$ network has a worse effect than $S V M$ with $F O A$, the reason may lie in the insufficient training samples for network in model training.

\section{ACKNOWLEDGMENT}

The authors declare no conflict of interest in preparing this article. We like to acknowledge the support from the National Natural Science Foundation of China (Grant No. 51541506, 51275524).

\section{REFERENCES}

1 Vanraj, Goyal, Deepam, Saini, Abhineet, et. al. "Intelligent Predictive Maintenance of Dynamic Systems Using Condition Monitoring and Signal Processing Techniques-A Review" (presentation, International Con- ference on Advances in Computing, Communication, \& Automation, Dehradun, India, September 2016), https://dx.doi.org/10.1109/ICACCA.2016.7578870.

2 Xiao, Yancai, Kang, Na, Hong, Yi, et. al. "Misalignment Fault Diagnosis of DFWT Based on IEMD Energy Entropy and PSO-SVM," Entropy 19, no. 1 (2017): 6-12, https://dx.doi.org/10.33390/e19010006

3 Lu, Chuanqi, Wang, Shaoping, and Tomovic, Mileta. "Fault Severity Recognition of Hydraulic Piston Pumps Based on EMD and Feature Energy Entropy" (presentation, Industrial Electronics and Applications, Auckland, New Zealand, June 2015), https://dx.doi.org/10.1109/ICIEA.2015.7334162.

4 Koehoorn, Joost, Sobiecki, André, Boda, Daniel, et. al. "Mathematical Morphology and Its Applications to Signal and Image Processing," 12th Inter- 
national Symposium 7110, no. 3 (2016): 648-654, https://dx.doi.org/10.1007/978-3-319-18720-4_2.

5 Wang, Hui and Li, Qi. "Signal Processing Based on Mathematical Morphology," Applied Mechanics and Materials 462-463, no. 1 (2014): 138-144, https://dx.doi.org/10.4028/www.scientific.net/AMM.462463.280 .

${ }^{6}$ Shih, Huang-Chia and Liu, En-Rui. "Automatic Reference Color Selection for Adaptive Mathematical Morphology and Application in Image Segmentation," IEEE Transactions on Image Processing 25, no. 10 (2016): 4665-4676, https://dx.doi.org/10.1109/TIP.2016.2586658.

7 Duan, Xian-Zhi, Ding, Ya-Jun, Qian, Sheng-You, et. al. "A Method of Image Segmentation Based on Combination of Improved Fast ICA and Mathematical Morphology," Microelectronics \& Computer 32 no. 2 (2015): 80-83, url: http://en.cnki.com.cn/Article_en/CJFDTOTALWXYJ201502019.htm.

${ }^{8}$ Li, Hua, Elmoataz, Abderrahim, Fadili, Jaral M., et. Al. "An Improved Image Segmentation Approach Based on Level Set and Mathematical Morphology," Proceedings of SPIE The International Society for Optical Engineering 5286, no. 2 (2015): 234-239, https://dx.doi.org/10.1117/12.538710.

${ }^{9}$ Chen, Qiong, Chen, Zhaowen, Sun, Wei, et. al. "A New Structuring Element for Multi-Scale Morphology Analysis and Its Application in Rolling Element Bearing Fault Diagnosis," Journal of Vibration \& Control 21, no. 4 (2013): 765-789, https://dx.doi.org/10.1177/1077546313486163.

${ }^{10} \mathrm{Li}$, Yongbo, Xu, Minqiang, Liang, Xihui, et. al. “Application of Bandwidth EMD and Adaptive MultiScale Morphology Analysis for Incipient Fault Diagnosis of Rolling Bearings," IEEE Transactions on Industrial Electronics 64, no. 8 (2017): 1-12, https://dx.doi.org/10.1109/TIE.2017.2650873.

${ }^{11} \mathrm{Lu}$, Chen, Wang, Zhen-Ya, Qin, Wei-Li, et. al. "Fault Diagnosis of Rotary Machinery Components Using a Stacked Denoising Autoencoder-Based Health State Identification," Signal Processing 130 (2016): 377-388, https://dx.doi.org/10.1016/l.sigpro.2016.01.028.

12 Vapnik, Vladimir. The Nature of Statistical Learning Theory. Springerville: Springer Science \& Business Media, 2013.

13 Yu, W'ang, Zhao, Jianghai, amd Wang, Deji. "Network Fault Diagnosis of SVM Based on Information Geometry," (presentation, International Conference on Progress in Informatics and Computing, Shanghai, China, June 2017), https://dx.doi.org/10.1109/PIC.2016.7949574.
${ }^{14}$ Zhang, Hai-Gang, Zhang, Sen, and Yin, Yi-Xin. "Multiclass Fault Diagnosis of BF Based on Global Optimization LS-SVM," Chinese Journal of Engineering 39, no. 1 (2016): 39-47, https://dx.doi.org/10.13374/j.issn20959389.2017.01.005.

15 Cai, Chengtao, Zhang, Chuanbin, Liu, Gang. "A Novel Fault Diagnosis Approach Combining SVM with Association Rule Mining for Ship Diesel Engine," (presentation, IEEE International Conference on Information and Automation, Ningbo, China, August 2016), https://dx.doi.org/10.1109/ICInfA.20167831809.

16 Zhang, Lijun, Xu, Jinwu, Yang, Jianhong, et. Al. "Multiscale Morphology Analysis and Its Application to Fault Diagnosis," Mechanical Systems and Signal Processing 22, no. 3 (2008): 597-610, https://dx.doi.org/10.1016/j.ymssp.2007.09.010.

${ }^{17}$ Pan, Wen-Tsao. "A New Fruit Fly Optimization Algorithm: Taking the Financial Distress Model as an Example," Knowledge-Based Systems 26 (2012): 69-74, https://dx.doi.org/10.1016/j.knosys.2011.07.001.

18 Tian, Xu and Li, Jie. "An Improved Fruit Fly Optimization Algorithm and Its Application in Aerodynamic Optimization Design," acta Aeronautica Et Astronautica Sinica 38, no. 4 (2017): 230-239, https://dx.doi.org/10.7527/S10006893.2016.0198.

19 Wu, Lianghong, Zuo, Cili, Zhang, Hongqiang, et. al.(2017). "Bimodal Fruit Fly Optimization Algorithm Based on Cloud Model Learning," Soft Computing 21, no. 7 (2015): 1-17, https://dx.doi.org/101007/s00500-015-1890-3.

${ }^{20}$ Smith, Wade A. and Randall, Robert B. "Rolling Element Bearing Diagnostics Using the Case Western Reserve University Data: A Benchmark Study," Mechanical Systems \& Signal Processing 64-65 (2015): 100-131, https://dx.doi.org/10.1016/j.ymssp.2015.04.021.

21 Wang, Dong, Sun, Shilong, and Tse, Peter W. "A General Sequential Monte Carlo Method Based Optimal Wavelet Filter: A Bayesian Approach for Extracting Bearing Fault Features," Mechanical Systems \& Signal Processing 52-53 (2015): 293-308, https://dx.doi.org/10.1016/j.ymssp.2014.07.005. 